\title{
ESTUDIO DE LOS INSTRUMENTOS NORMATIVOS DE ORDENAMIENTO AMBIENTAL DEL MUNICIPIO DE TURBO (ANTIOQUIA), EN EL MARCO DE LAS POLÍTICAS DE ORDENAMIENTO AMBIENTAL DE COLOMBIA
}

\author{
Dayro Alberto Acevedo Ortiz ${ }^{\underline{1}}$ \\ Gloria Yaneth Flórez Yepes ${ }^{2}$ \\ Recibido el 10 de noviembre de 2014, aprobado el 17 de junio de 2015 y \\ actualizado el 04 noviembre de 2015
}

DOI: 10.17151/luaz.2016.42.11

\section{RESUMEN}

El municipio de Turbo (Antioquia, Colombia) cuenta con una gran riqueza ecosistémica, y asociado a esto y a las políticas y normativas nacionales, las autoridades ambientales han construido diferentes instrumentos de ordenamiento ambiental, formulados con distintos marcos de política de ordenamiento con disímiles metodologías (escalas cartográficas, normatividad y enfoques metodológicos), lo que ha causado incertidumbre sobre la articulación, complementariedad, armonización e integralidad de los instrumentos, en los ámbitos territorial, de la protección de los ecosistemas y de la promoción del desarrollo sostenible.

Este artículo hace parte de uno de los resultados de investigación de un trabajo de grado de Maestría, cuyo objetivo fue analizar cómo los instrumentos de ordenamiento ambiental en el contexto territorial (físico-espacial), se cruzan y se afectan positiva o negativamente a partir de las políticas y normativas de ordenamiento ambiental y cómo dichas políticas inciden sobre el ordenamiento de los ecosistemas del municipio de Turbo. Metodológicamente el proyecto se desarrolla bajo un enfoque mixto, abordando ocho instrumentos de ordenamiento ambiental, utilizando técnicas como fichas resumen, reuniones con expertos utilizando la técnica de Delphi y la escala de Likert para la aplicación de la encuesta, igualmente se utilizaron los sistemas de información geográfica con el fin de realizar los análisis espaciales, y se elaboró un instrumento comparativo que permitiera generar el análisis. El resultado principal que se muestra en este artículo es un análisis del papel que han jugado las autoridades ambientales nacionales y regionales en la articulación de los instrumentos de ordenamiento ambiental municipal; y como principal conclusión se obtuvo que las principales falencias de los instrumentos de ordenamiento ambiental del municipio de Turbo, tienen su génesis en la forma de abordaje de la planificación y formulación de política pública ambiental desde las autoridades ambientales, así como de dictar regulaciones, donde a nivel del SINA no existe unidad conceptual, de escala ni de enfoque en el diseño y orientación de la política pública ambiental en su relación con el territorio, lo que evidencia grietas conceptuales y formas diversas desarticuladas de planificación del desarrollo y del quehacer de lo ambiental.

\section{PALABRAS CLAVE}

Ordenamiento ambiental, desarrollo sostenible, territorio, ecosistemas estratégicos. 


\title{
STUDY OF REGULATORY INSTRUMENTS OF ENVIRONMENTAL MANAGEMENT OF THE MUNICIPALITY OF Turbo (Antioquia), UNDER THE ENVIRONMENTAL MANAGEMENT POLICIES OF COLOMBIA
}

\begin{abstract}
The municipality of Turbo (Antioquia, Colombia) has a great ecosystemic richness and associated with this, and the national policies and regulations, environmental authorities have built various instruments for environmental management, formulated with different policy framework systems with dissimilar methodologies (map scales, normative and methodological approaches), which has caused uncertainty about the articulation, complementarity, harmonization and comprehensiveness of the instruments in the territorial, protection of ecosystems and sustainable development promotion fields.
\end{abstract}

This article is part of one of the results of a Master's degree requirement research project aimed at analyzing how the environmental management instruments in the territorial context (physical-spatial) intersect and affect each other positively or negatively from the environmental management policies and regulations and how these policies have an impact on the ecosystems in the Municipality of Turbo, Antioquia. Methodologically the project was developed under a mixed approach, addressing eight instruments for environmental management and using techniques such as summary cards, meetings with experts using the Delphi technique and Likert's scale for the application of the survey. Also the geographic information systems were used to perform spatial analysis and a comparative instrument to generate the analysis was developed. The main result shown in this article is an analysis of the role played by national and regional environmental authorities in articulating municipal environmental management instruments. The main conclusion obtained was that the focal shortcomings of the instruments of environmental management in the municipality of Turbo, have their origin in the way planning and formulation of environmental public policy is approached from environmental authorities, as well as in issuing regulations since at SINA level there is no conceptual unity of the scale or approach on the design and orientation of public environmental policy in relation to the territory, which shows conceptual cracks and various disjointed forms for development planning and of the environmental scope.

KEY WORDS: Environmental planning, sustainable development, territory, strategic ecosystems.

\section{INTRODUCCIÓN}

El municipio de Turbo, altamente estratégico por sus ecosistemas y biodiversidad (Corpourabá, 2012), se localiza en el noroccidente del departamento de Antioquia, forma parte de la región del Urabá antioqueño y se ubica dentro del Chocó biográfico (Municipio de Turbo, 2008). Tiene un área de $3.055 \mathrm{~km}^{2}$ (el más grande del departamento de Antioquia), de los cuales $11,9 \mathrm{~km}^{2}$ hacen parte del área urbana y los $3.043,1 \mathrm{~km}^{2}$ restantes corresponden al área rural (Gobernación de Antioquia, 2011).

Dentro de los diferentes estudios realizados en la región de Urabá y del departamento de Antioquia relacionados con el ordenamiento ambiental, se han encontrado entre otros los siguientes: Plan Estratégico de Urabá 2011-2020 (Comisión Tripartita, 2009), Plan de Gestión Ambiental Regional 2012-2024 (Corpourabá, 2012), Plan de Ordenamiento Territorial del municipio de Turbo 
(Municipio de Turbo, 2012), Lineamientos y Estrategias de Manejo Integrado de la Unidad Ambiental Costera del Darién (Gobernación de Antioquia, 2008), Estado de los recursos naturales y del medio ambiente de Antioquia (Gobernación de Antioquia, 2008), Ecosistemas Estratégicos del Departamento de Antioquia (Gobernación de Antioquia, 2005); si bien es cierto que éstos abordan los diferentes estudios e instrumentos de planificación ambiental para el municipio de Turbo y en ocasiones para toda la zona de Urabá, no se estudian a profundidad los componentes ambientales de los instrumentos, ni se analizan las zonas ni las relaciones de traslape entre los mismos, a la vez que no se investigan cómo las diferentes políticas de ordenamiento ambiental se aplican o inciden sobre los instrumentos municipales de ordenamiento ambiental.

Se parte entonces del concepto de territorio, el cual tiene diferentes acepciones y es utilizado por diversas ciencias y disciplinas, bajo disímiles enfoques teóricos y metodológicos, para este caso se consideraron los enfoques asumidos por Restrepo (2011) y Toro (2012) quienes interpretan al territorio como el espacio donde debe haber un entendimiento de relaciones entre las personas y los recursos naturales que las rodean.

Otro concepto importante en el abordaje de esta investigación fue el de ordenamiento ambiental, el cual lo plantea Gallo (2012, p.29) como:

[...] el proceso político y técnico, en cabeza del Estado y que comprometa corresponsablemente a todos los actores del desarrollo del territorio, orientado a soportar y articular los procesos de toma de decisión y actuación sobre el territorio en sus diferentes escalas, para prevenir, solucionar o reversar los conflictos.

Asimismo, Cabanzo y Fracasso (1997, p.36) consideran que el ordenamiento ambiental se debe centrar básicamente en "el equilibrio entre transformación, conservación y restauración de los ecosistemas cuando hayan sido perturbados más allá de su capacidad de resiliencia". Motta (2005) menciona que el ordenamiento ambiental ha sido identificado y enmarcado con la definición de usos de la tierra compatibles con las condiciones ambientales y el manejo integral de las cuencas hidrográficas, áreas de manejo especial y ecosistemas estratégicos, donde se cuente con la efectiva participación de las comunidades y actores existentes en el territorio.

Ahora bien, no se puede hablar de ordenamiento ambiental sin considerar la Ley Orgánica de Ordenamiento Territorial cuyo objetivo es:

[...] dictar las normas orgánicas para la organización político-administrativa del territorio colombiano; enmarcar en las mismas el ejercicio de la actividad legislativa en materia de normas y disposiciones de carácter orgánico relativas a la organización político-administrativa del Estado en el territorio; establecer los principios rectores del ordenamiento; definir el marco institucional e instrumentos para el desarrollo territorial; definir competencias en materia de ordenamiento territorial entre la Nación, las entidades territoriales y las áreas metropolitanas y establecer las normas generales para la organización territorial. (Ministerio del Interior y de Justicia, 2011, p. 13)

En este sentido, los instrumentos utilizados están enmarcados dentro del concepto de la Ley Orgánica de Ordenamiento Territorial.

Otro aspecto importante dentro de las políticas de ordenamiento ambiental es la Política Nacional de Biodiversidad (PNB), la cual fue creada en Colombia en 1996, 
esfuerzo que es complementado con la Propuesta Técnica para la Formulación de un Plan de Acción Nacional en Biodiversidad: Colombia biodiversidad Siglo XXI (1998), que ha sido una guía para el trabajo institucional para conservar y valorar su biodiversidad, con el fin de prevenir y controlar su acelerada pérdida y transformación, así como para reducir y mitigar los efectos negativos que esto genera sobre la calidad de vida (Ministerio de Ambiente y Desarrollo Sostenible, 2011).

Ahora bien, la aplicación de políticas y la gestión de las mismas que busquen dentro del territorio el equilibrio propuesto por el ordenamiento ambiental y el concepto de desarrollo sostenible, entre otros, se entiende por territorialización, esta implica tener en los mismos niveles de importancia las dimensiones sociocultural, económica, natural y político-institucional en las decisiones sobre el territorio en materia de ordenamiento y gestión territorial (Gutiérrez, 2012).

De acuerdo con lo anterior, en esta investigación se buscó lograr nexos entre lo teórico y lo práctico (este nexo entre lo teórico y práctico se refiere a lo escrito en los planes utilizados como instrumentos y lo analizado a partir de SIG) y aportar elementos al análisis del ordenamiento ambiental territorial del municipio de Turbo (Antioquia), que facilite visualizar la relación y armonización de los instrumentos normativos de ordenamiento ambiental, además de la incidencia que tienen las políticas de ordenamiento ambiental en la aplicación de los instrumentos.

En este sentido, en esta investigación metodológicamente lo que hizo fue hacer una comparación de los instrumentos normativos relacionados con los planes estratégicos, planes de ordenamiento territorial, lineamientos estratégicos de manejo, entre otros, para ver cómo afectan e inciden sobre el ordenamiento de los ecosistemas del municipio de Turbo, lo cual fue abordado con instrumentos de recolección de datos como entrevistas con expertos, sistemas de información geográfica y matrices de análisis.

Finalmente, con esta investigación a partir del análisis comparativo de la coherencia que existe entre los instrumentos normativos de ordenamiento ambiental, se generan elementos de análisis para que las instituciones nacionales, regionales y locales encargadas de formular las políticas ambientales puedan tener coherencia y articulación en la formulación de los instrumentos de ordenamiento, con el fin de optimizar esfuerzos, evitar contradicciones, hacer más eficiente la información y lograr un mejor proceso de planificación futura.

\section{MATERIALES Y MÉTODO}

Desde el punto de vista epistemológico, el objeto de la investigación integra componentes socioambientales, los cuales se fusionan bajo el constructo teórico de "ordenamiento ambiental", para soportar el discurso científico toma elementos de teorías de las ciencias sociales y naturales, además de la disciplina jurídica.

El tipo de investigación es descriptivo y analítico, bajo la perspectiva documental, toda vez que parte del análisis teórico y espacial de la información; se complementa a través del uso de bases de datos y la consulta a expertos, mediante el método Delphi, para poder llegar a la comprobación de los supuestos planteados en la investigación. 


\section{Unidad de análisis y población}

La unidad de análisis de la presente investigación se compuso del total de los instrumentos de ordenamiento ambiental del municipio de Turbo (los cuales en la actualidad son ocho), en este sentido no se cuenta con muestra probabilística y la unidad de análisis es igual a la población o universo.

\section{Técnicas de investigación}

A nivel de las técnicas de investigación para capturar la información, se utilizaron:

\section{Fichas resumen}

Se realizó la revisión de información secundaria consignada en los estudios, planes de ordenamiento ambiental y bases de datos cartográficas que tienen la autoridad ambiental regional Corpourabá, el municipio y demás instituciones. Para la organización de la información secundaria de textos se utilizaron fichas resumen para textos y cartográfica, con las que se recogió y sistematizó la información. Las fichas resumen contenían la institución o autoridad ambiental, el contenido de cada instrumento normativo de ordenamiento con un breve resumen y la cartografía existente.

\section{Entrevistas con expertos}

Se realizó la entrevista con siete expertos representantes de entidades e institutos de investigación con conocimiento y/o experiencia en el tema de ordenamiento territorial involucrando conceptos relacionados con el marco metodológico de actuación nacional, desde la praxis de los instrumentos del SINA, entre otros, para ello se aplicó el método Delphi, el cual es una técnica utilizada para la evaluación de programas y toma de decisiones, que recoge información de diversos expertos o especialistas en el tema, los cuales no se pueden reunir, pero que busca generar consensos o tendencias (Landeta, 1999).

Liston y Turoff citado por Landeta (1999), conciben la técnica Delphi como un método de estructuración de un proceso de comunicación grupal de personas expertas, que es efectivo cuando se trata de analizar un problema complejo.

\section{Instrumento}

El instrumento que se sometió a validación por el grupo de expertos, se formuló teniendo en cuenta los objetivos específicos, en los cuales se trabajaron las variables: Instrumento de ordenamiento ambiental y Políticas de ordenamiento. A su vez, de las variables se desprendieron 10 categorías de análisis, sobre las cuales se conformaron las preguntas de la primera versión del cuestionario utilizado, y sobre el análisis de los resultados se construyó la segunda versión del cuestionario constituido por 12 preguntas; a partir de los resultados de la segunda versión del cuestionario se realizó el análisis final de la información.

Los instrumentos de ordenación ambiental son aquellos que han sido emitidos por varias autoridades ambientales con el fin de conservar y manejar sosteniblemente los ecosistemas, para este caso: Declaración de Reserva Forestal Protectora de los humedales localizados entre los ríos León y Suriquí, Declaración de Parque Nacional Natural los Katíos; Política Marino-Costera; Plan de Ordenación Forestal de Urabá; Reservas municipales Cerro El Cuchillo y Lomas aisladas; Plan de Manejo Integrado de los humedales del Bajo y Medio Atrato; Zonificación y plan de acción para las zonas de conservación y recuperación de los manglares del golfo 
de Urabá y mar Caribe antioqueño; Plan de Ordenamiento y Manejo de la Cuenca hidrográfica del río Turbo.

La política de ordenamiento ambiental se consideró como aquellos lineamientos generales para definir las regulaciones cuyo objetivo es la protección de determinados ecosistemas, tales como Planes de ordenamiento ambiental, Políticas de Política Nacional para la Gestión Integral de la Biodiversidad y sus Servicios Ecosistémicos, entre otros.

En la elaboración y aplicación de cuestionarios, se buscó que las respuestas fueran cuantificadas y ponderadas, categorizando los consultados.

A partir de los antecedentes recogidos en el marco teórico y de la información secundaria cotejada (normativas, zonificaciones, cartografías y políticas, entre otros), se establecieron las ideas o tópicos más determinantes que debían ser analizados en el trabajo, de allí que en el marco de los objetivos específicos, se confeccionó una lista de categorías, las cuales se relacionan a continuación:

Tabla 1. Variables y categorías de análisis consideradas en el proyecto

\begin{tabular}{|c|c|c|c|c|}
\hline Objetivo & Variable & Categoria & $\begin{array}{l}\text { Preguntas Primer } \\
\text { Cuestionario } \\
\text { (abiertas) }\end{array}$ & $\begin{array}{l}\text { Preguntas Segundo } \\
\text { Cuestionario } \\
\text { (escala Likert) }\end{array}$ \\
\hline $\begin{array}{l}\text { Analizar el } \\
\text { papel que } \\
\text { han jugado } \\
\text { las } \\
\text { autoridades } \\
\text { ambientales } \\
\text { nacionales y } \\
\text { regionales en } \\
\text { la articulación } \\
\text { de los } \\
\text { instrumentos } \\
\text { de } \\
\text { ordenamiento } \\
\text { ambiental } \\
\text { municipal }\end{array}$ & $\begin{array}{l}\text { Politicas de } \\
\text { ordenamiento }\end{array}$ & $\begin{array}{l}\text { Articulacion de } \\
\text { los instrumentos } \\
\text { de planificación }\end{array}$ & $\begin{array}{l}\text { ¿Usted cree que } \\
\text { existe un marco } \\
\text { metodológico de } \\
\text { actuaciónnacional de } \\
\text { ordenamiento } \\
\text { ambiental territorial, } \\
\text { que identifique } \\
\text { objetivos y temáticas } \\
\text { en el municipio de } \\
\text { Turbo? } \\
\text { Según su opinion } \\
\text { desde el punto de } \\
\text { vista normativo, ¿el } \\
\text { ordenamiento y } \\
\text { territorial } \\
\text { fundamentalmente } \\
\text { ambiental } \\
\text { transversal? }\end{array}$ & $\begin{array}{l}\text { Las dependencias } \\
\text { del Ministerio de } \\
\text { Ambiente se } \\
\text { articulany coordinan } \\
\text { frente } \\
\text { ordenamiento al } \\
\text { ambiental local y } \\
\text { regional } \\
\text { El ordenamiento } \\
\text { territorial } \\
\text { esencialmente } \\
\text { ambiental } \\
\text { transversal }\end{array}$ \\
\hline
\end{tabular}




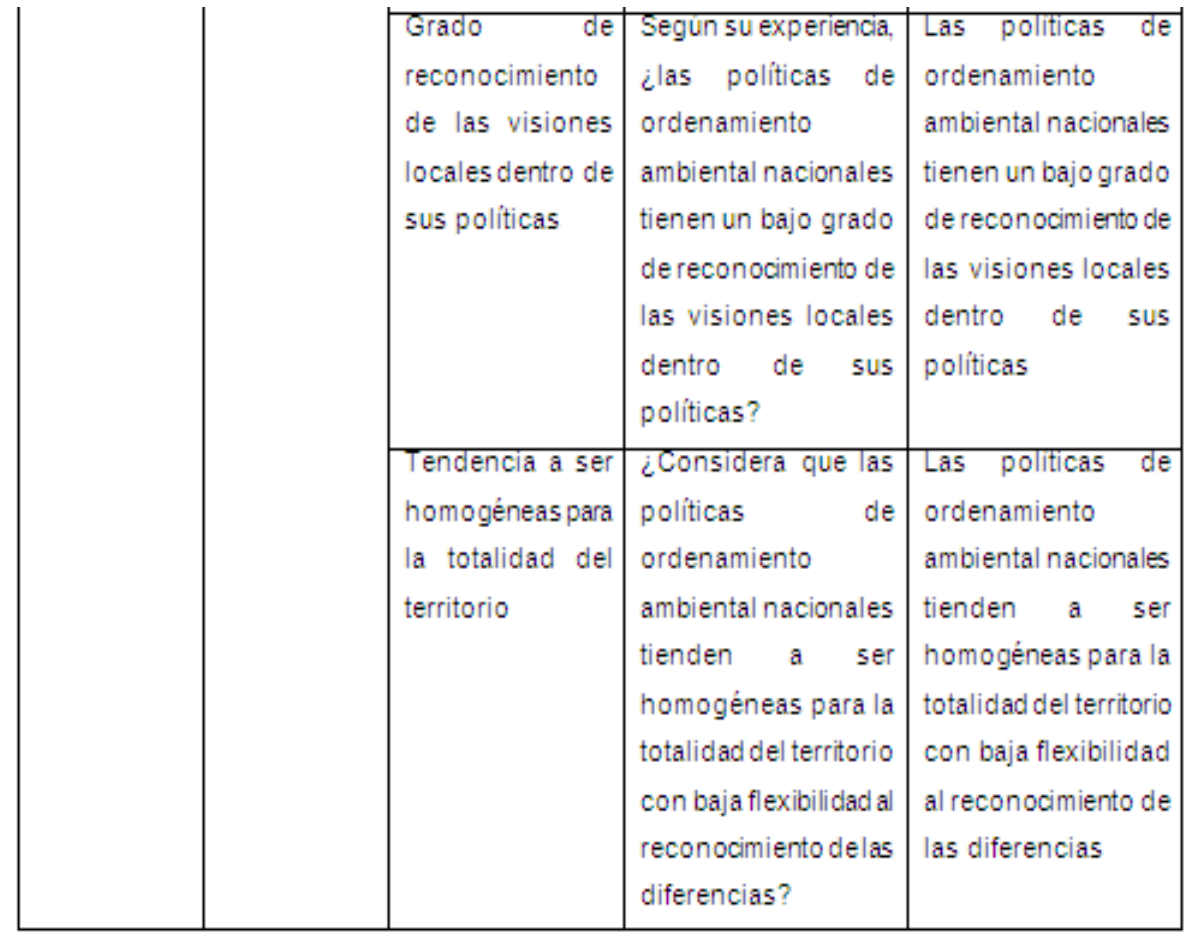

\section{Fase preliminar}

En esta fase se seleccionaron los expertos, a los cuales se aplicó la técnica Delphi. La muestra que se trabajó fue de siete personas expertas, representantes de entidades e institutos de investigación con conocimiento y/o experiencia en el tema (dos de Corpourabá, uno del municipio de Turbo, uno de la Universidad de Antioquia, uno del Ministerio de Ambiente y Desarrollo Sostenible, uno de la Unidad de Parques Naturales Nacionales y uno de la Gobernación de Antioquia); las personas se seleccionaron de una manera no aleatoria, pues de antemano se sabía lo que se estaba buscando de la información. La muestra compuesta por siete expertos responde a las consideraciones de Astigarraga (2003), que plantea que el número menor recomendable es de siete peritos, además esta cantidad se tomó por la dificultad de encontrar personas versadas y especialistas en el tema que pudieran y estuvieran en condiciones de colaborar con los cuestionarios.

En la fase preliminar se formuló la primera versión del cuestionario conformado por 10 preguntas abiertas.

\section{Fase exploratoria}

Se realizó la aplicación del cuestionario (primera ronda), con las respuestas de los expertos se realizó el análisis, las cuales por ser abiertas se trabajaron bajo medidas de tendencia central (moda y mediana).

En la aplicación de la primera ronda del cuestionario se presentaron las siguientes situaciones:

- Se presentaron errores de redacción que dificultaron el entendimiento de las preguntas.

- Se recomendó por los expertos, para la segunda versión del cuestionario, incluir dos nuevos tópicos (articulación de las dependencias del MADS frente al 
ordenamiento local y dispersión e instancias y espacios en los ordenamientos sectoriales) y no incluir la pregunta: ¿Considera que el marco legal que soporta los instrumentos de ordenamiento ambiental local existente en el municipio de Turbo permite la adecuada armonización de los instrumentos en el territorio?, esto por la generalidad de la apreciación y porque está integrado en otras preguntas.

\section{Fase final}

A partir de las recomendaciones y de las respuestas de expertos se generó la segunda versión del cuestionario con 12 preguntas, utilizando una escala Likert. Se aplicó el cuestionario sobre los siete expertos.

En la sistematización y análisis de la información se trataron las respuestas en términos porcentuales identificando las tendencias de los consultados y ubicándolos en una categoría.

Finalmente se presentó a los expertos los resultados del análisis de la información estadística.

\section{RESULTADOS}

Análisis del papel jugado por las autoridades ambientales nacionales y regionales en la articulación de los instrumentos de ordenamiento ambiental de Turbo

En el análisis de la normativa y la política ambiental colombiana, se evidencia que la competencia del Ministerio de Ambiente y Desarrollo Sostenible en el marco del ordenamiento ambiental del territorio, incide de forma integral en la manera en que se planifica el desarrollo socioeconómico en el territorio, cualquiera sea el nivel en el que se realice, lo cual está soportado en la Ley 99 de 1993 y el Decreto Ley 3570 de 2011, los cuales le dan al Ministerio de Ambiente y Desarrollo Sostenible dos tipos de funciones asociadas al ordenamiento ambiental del territorio, que determinan igualmente, como consecuencia lógica, la manera que tienen las Corporaciones autónomas y de desarrollo sostenible y las autoridades ambientales urbanas de incidir en el ordenamiento del territorio.

El primer grupo o categoría de atributos, que por demás ejerce de manera habitual el Ministerio de Ambiente y Desarrollo Sostenible, consiste en expedir regulaciones e instrumentos de planificación de determinados ecosistemas estratégicos, como también de los recursos naturales renovables (Congreso de Colombia, 1993); así se tienen, por ejemplo, los instrumentos de planificación del manejo de las áreas protegidas nacionales, sus actos de declaratoria, las regulaciones especiales sobre páramos, humedales y manglares, también son expresiones de esta forma de abordar el ordenamiento ambiental del territorio, las vedas de especies forestales para su aprovechamiento o de especies animales para su caza (Ministerio de Ambiente, Vivienda y Desarrollo Territorial, 2011).

Por su parte, las Corporaciones Autónomas Regionales y de Desarrollo Sostenible como ejecutoras de la política pública ambiental, y dentro de esas Corpourabá, han traducido el ejercicio de esta misma línea de gestión restringiendo su ámbito de intervención en el ordenamiento territorial a dos tipos de procesos: la formulación de determinantes de los Planes de Ordenamiento Territorial y la concertación de asuntos ambientales de los mismos (Congreso de Colombia, 1997), además de las funciones que les da la Ley 99 de 1993. Así como las regulaciones expedidas por el Ministerio de Ambiente y Desarrollo Sostenible, 
respecto de ciertos ecosistemas de importancia: las cuencas hidrográficas, los humedales, los páramos, los manglares, las áreas protegidas, las reservas forestales, entre otras (Congreso de Colombia, 1993). En este sentido, Corpourabá en el territorio de Turbo ha formulado los planes: el POMCA del río Turbo, el Plan de Manejo del Manglar, la Unidad Ambiental Costera del Darién, el Plan de Manejo de los Humedales del río Atrato, la Reserva forestal regional del Suriquí, entre otros.

Un segundo grupo de atribuciones otorgadas por la Ley 99 de 1993 y el Decreto Ley 3570 de 2011 al Ministerio de Ambiente y Desarrollo Sostenible, son las que le permiten dictar lineamientos y directrices de regulación y política para la planificación del desarrollo y la formulación de la política pública sectorial (Ministerio de Ambiente, Vivienda y Desarrollo Territorial, 2011), de manera que se asegure la inserción de aspectos asociados al ordenamiento ambiental del territorio en todos los ámbitos de la planificación pública en el país (Congreso de Colombia, 1993).

El ordenamiento ambiental del territorio es una función pública (Betancur, 1999), que involucra al Estado en su conjunto (Ministerio del Medio Ambiente, 1998), es decir, que trasciende de lo que habitualmente se denomina el "sector ambiental", por lo que resulta ser un propósito estatal transversal a los diversos sectores del desarrollo económico, y respecto del cual, el Ministerio de Ambiente y Desarrollo Sostenible tiene unas funciones definidas en la Ley 99 de 1993, así como en el Decreto 3570 de 2011.

Ahora bien, el artículo $5^{\circ}$ de la Ley 99 de 1993, asigna al Ministerio de Ambiente y Desarrollo Sostenible tres funciones regentes en materia de ordenamiento ambiental:

- $\quad$ Establecer las reglas y criterios de ordenamiento ambiental de uso del territorio y de los mares adyacentes, para asegurar el aprovechamiento sostenible de los recursos naturales renovables y del medio ambiente;

Preparar, con la asesoría del Departamento Nacional de Planeación, los planes, programas y proyectos que en relación con el ordenamiento ambiental del territorio, deban incorporarse a los proyectos del Plan Nacional de Desarrollo del Plan Nacional de Inversiones que el Gobierno someta a consideración del Congreso;

- $\quad$ Expedir y actualizar el estatuto de zonificación de uso adecuado del territorio para su apropiado ordenamiento y las regulaciones nacionales sobre el uso del suelo en lo concerniente a sus aspectos ambientales. (Congreso de Colombia, 1993, pp. 5-6).

Estas atribuciones son, además, reiteradas por el artículo $2^{\circ}$ del Decreto Ley 3570 de 2011, al definir entre otras funciones del Ministerio de Ambiente y Desarrollo Sostenible, las siguientes:

Diseñar y formular la política nacional en relación con el ambiente y los recursos naturales renovables, y establecer las reglas y criterios de ordenamiento ambiental de uso del territorio y de los mares adyacentes, para asegurar su conservación y el aprovechamiento sostenible de los recursos naturales renovables y del ambiente [...]

- Apoyar a los demás Ministerios y entidades estatales, en la formulación de las políticas públicas, de competencia de los mismos, que 
tengan implicaciones de carácter ambiental y desarrollo sostenible y establecer los criterios ambientales que deben ser incorporados en esta formulación de las políticas sectoriales [...]

- Preparar, con la asesoría del Departamento Nacional de Planeación, los planes, programas y proyectos que en materia ambiental, o en relación con los recursos naturales renovables y el ordenamiento ambiental del territorio, deban incorporarse a los proyectos del Plan Nacional de Desarrollo y del Plan Nacional de Inversiones que el Gobierno someta a consideración del Congreso de la República. (Ministerio de Ambiente, Vivienda y Desarrollo Territorial, 2011, pp. 3-4)

Resulta claro que al Ministerio de Ambiente y Desarrollo Sostenible, además de que le corresponde dictar decisiones de ordenamiento ambiental del territorio respecto de ciertas áreas o ecosistemas estratégicos (cuencas hidrográficas, SINAP, humedales, manglares, páramos y zonas marinas), le compete formular lineamientos y criterios de ordenamiento ambiental, para direccionar a los sectores productivos, en materia del desarrollo sostenible.

No obstante lo anterior, de acuerdo a los expertos consultados, hay fragmentación de los procesos de formulación de política y programas sobre el territorio y se carece de instancias efectivas de articulación desde el sector ambiental a nivel local, en este sentido el $57,1 \%$ y el $28,6 \%$ de los expertos consideran respectivamente estar "muy en desacuerdo" y "en desacuerdo" cuando se les presenta la premisa "Los instrumentos de ordenamiento ambiental de Turbo se complementan y armonizan territorialmente" como se muestra en la Figura 1.

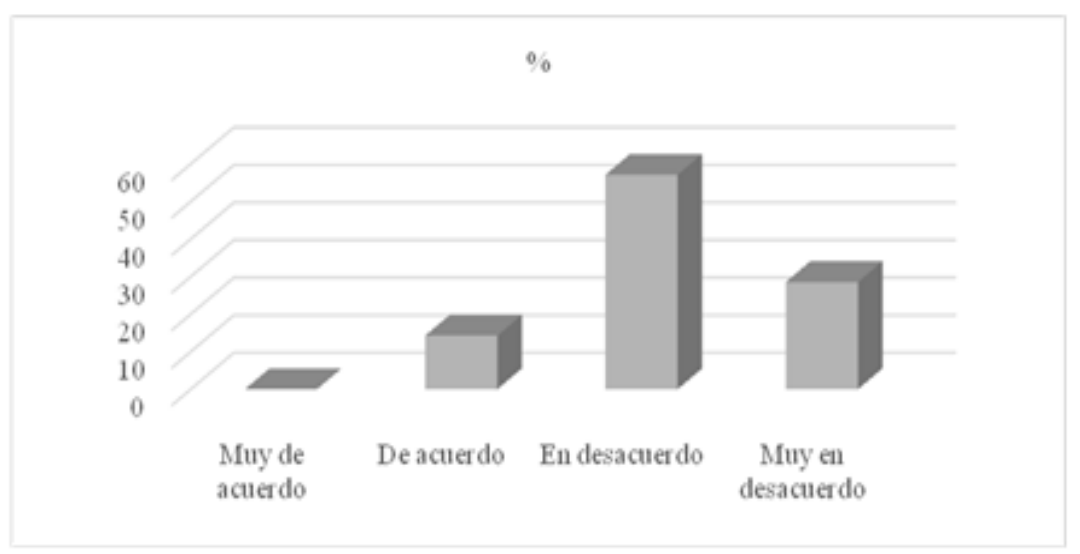

Figura 1. Pregunta a expertos sobre la premisa "Los instrumentos de

ordenamiento ambiental de Turbo se complementan y armonizan territorialmente".

"En Colombia se ha adoptado un criterio cada vez más dinámico para el establecimiento de objetivos de política ambiental internacional y se ha incorporado el medio ambiente en la estrategia de cooperación internacional del país", (Cepal, 2014. P.112) por otro lado, se han formulado políticas en diferentes áreas y con diversos enfoques y propósitos sobre el territorio, sumadas a los espacios de ordenamientos sectoriales (minero energéticos, viales, agrícola, entre otros), a través de decisiones que afectan el uso del suelo y las condiciones ambientales, tales como los proyectos de interés nacional, los derivados de las denominadas locomotoras del desarrollo o megaproyectos de infraestructura o productivos. Esto se traduce en desarticulación institucional en el territorio del municipio de Turbo, con multiplicidad de instrumentos de planeación, sin capacidad de armonización, desarticulación municipal entre los procesos de ordenamiento territorial y los derivados de la gestión ambiental. De allí que el 
$42,9 \%$ de los expertos consultados manifestaron estar "en desacuerdo" y el 28,6\% "muy en desacuerdo", cuando se les consultó la premisa "Existe un marco metodológico de actuación nacional de ordenamiento ambiental territorial, que identifique objetivos y temáticas en el municipio de Turbo" (Figura 2).

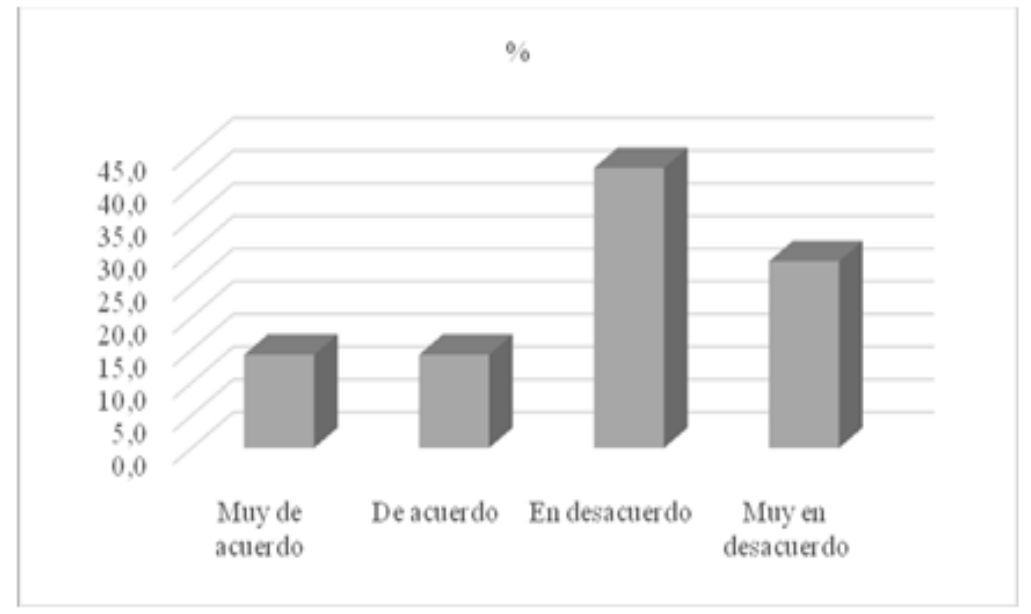

Figura 2. Pregunta a expertos "Existe un marco metodológico de actuación

nacional de ordenamiento ambiental territorial, que identifique objetivos y temáticas".

El Ministerio de Ambiente y Desarrollo Sostenible ha abordado desde tiempo atrás de manera fraccionada y segmentada por recursos naturales o ecosistemas, su quehacer frente al ordenamiento territorial, poniendo internamente en competencia directrices y enfoques metodológicos formulados desde cada dependencia. En este sentido los expertos consultados sobre la premisa "Las dependencias del Ministerio de Ambiente y Desarrollo Sostenible se articulan y coordinan frente al ordenamiento ambiental local y regional", consideran en un $42,9 \%$ estar "muy en desacuerdo" y el 28,6\% "en desacuerdo" (Figura 3).

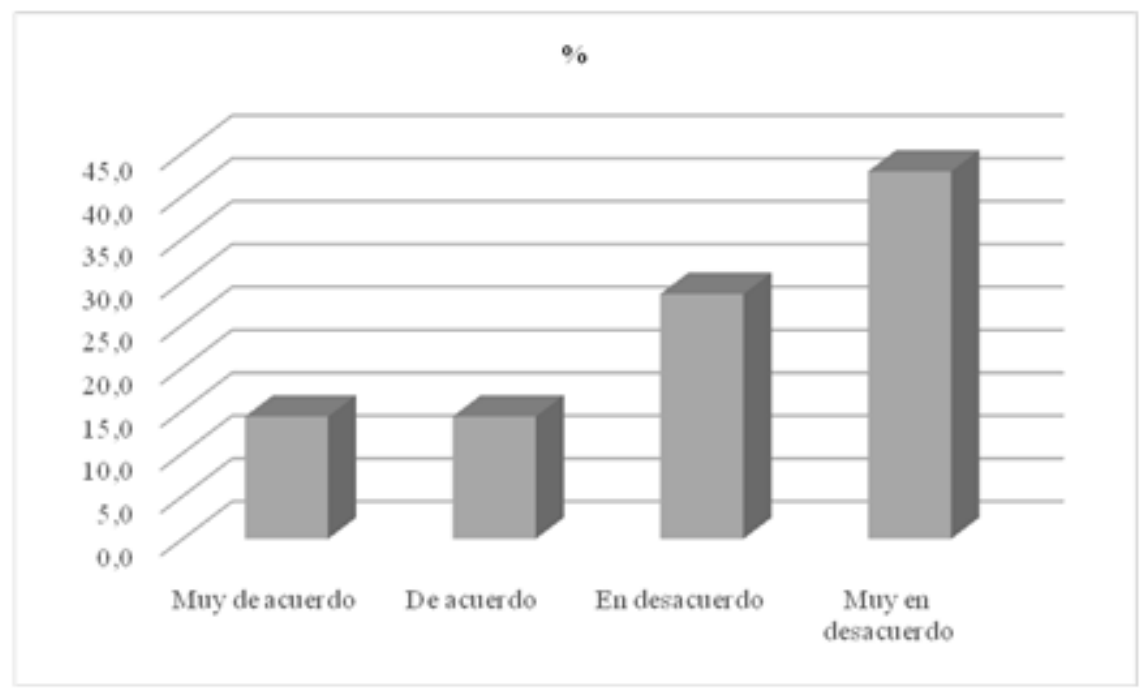

Figura 3. Pregunta a expertos sobre articulación local y regional de las

$$
\text { dependencias del MADS. }
$$

La fragmentación al interior del Ministerio de Ambiente, como rector de la política de ordenamiento ambiental, se observa desde el quehacer de las diferentes 
dependencias, como la Dirección de Bosques, Biodiversidad y Servicios Ecosistémicos, la cual realiza la clasificación del país en áreas forestales, protectoras o productoras; desde la Dirección de Recurso Hídrico, se planifica y formula política pública a partir de la cuenca como unidad de gestión y de planificación; desde Parques Nacionales Naturales se concibe el territorio y su planificación y ordenamiento ambiental a partir de categorías de áreas protegidas; desde la dirección de asuntos marinos se direcciona el ordenamiento ambiental en las zonas marino-costeras. Esto sin tener en cuenta las autoridades ambientales regionales: Corporaciones Autónomas regionales, departamentos, municipios, la DIMAR en zonas marinas, los resguardos indígenas y consejos comunitarios de Negritudes en cabeza del INCODER, entre otros.

De acuerdo a las respuestas de los expertos y el análisis de Sistema de Información Geográfico, los instrumentos de ordenamiento ambiental de Turbo difícilmente se complementan y no se armonizan, como se observó anteriormente en el aparte del análisis de la armonización físico-espacial entre los instrumentos normativos de ordenamiento ambiental territorial. En este sentido, el $100 \%$ de los expertos consultados consideran que existe en el país una alta dispersión de instancias y espacios en cada una de los ordenamientos sectoriales, en el ordenamiento territorial y en los instrumentos de planeación del desarrollo y del sector ambiental.

Desde la praxis de las entidades del SINA, en Turbo se cuenta con diversas metodologías para la valoración y monitoreo del territorio desde una visión ecosistémica y prospectiva, que no se armonizan para permitir la toma de decisiones desde el ordenamiento territorial la mayor parte de los expertos, consideran que en los procesos de ordenamiento ambiental del municipio de Turbo, existe abundancia y complejidad de categorías para el manejo del territorio, que dificultan las posibilidades de comprensión y manejo en escalas más amplias (Figura 4).

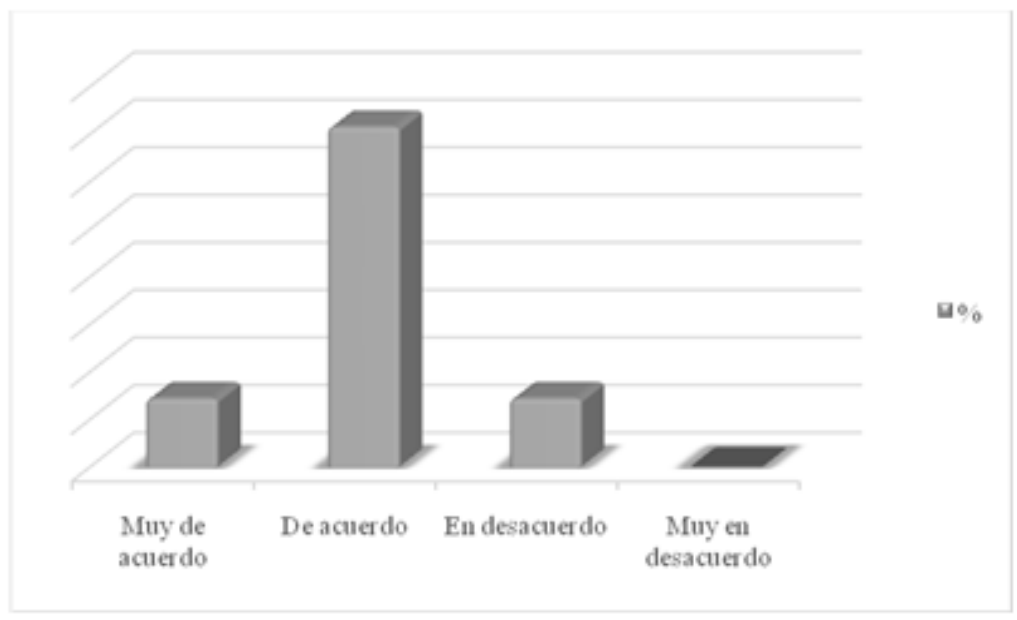

Figura 4. Pregunta a expertos sobre la existencia de profusión de categorías

Ante la existencia de numerosos categorías prevalecen aproximaciones desde la lógica del recurso natural, desarticuladas de las dinámicas socioeconómicas o por lo menos tibiamente integradas; de allí que los expertos consultados creen en un $71,4 \%$ que en el territorio de Turbo, desde la praxis de las entidades del SINA los instrumentos de ordenamiento ambiental no se encuentran articulados a las dinámicas socioeconómicas. 
Complementariamente a lo anterior, se evidencia que no existe un marco metodológico de actuación nacional, que identifique objetivos y temáticas concretas sobre las cuales actuar, dependiendo del tipo de situación a resolver en cada una de las escalas territoriales. Apreciación que es corroborada por los expertos, los cuales en un $71,4 \%$ responden que no hay claridad en las categorías derivadas de las legislaciones sectoriales, en cuanto al uso del suelo y frente a condiciones ambientales a su interior, e igualmente en un $85,7 \%$ los expertos manifiestan que las políticas de ordenamiento ambiental nacionales tienen un bajo grado de reconocimiento de las visiones locales, lo que genera conflictos en el territorio de Turbo.

En este sentido, desde diferentes fuentes gubernamentales se han diseñado normativas, políticas, directrices, planes de manejo de ecosistemas, entre otros, que han definido categorías que expresan decisiones de ordenamiento ambiental en Turbo y que no cuentan con un marco claro en cuanto a alcance, escalas y régimen de usos aplicables.

De allí que el $71,4 \%$ de los expertos consultados indican estar en "desacuerdo" con la tesis: "El Estado Colombiano tiene un marco instrumental que permita la articulación de los instrumentos de planificación sectorial en relación con el territorio de Turbo y sus condiciones ambientales" (Figura 5).

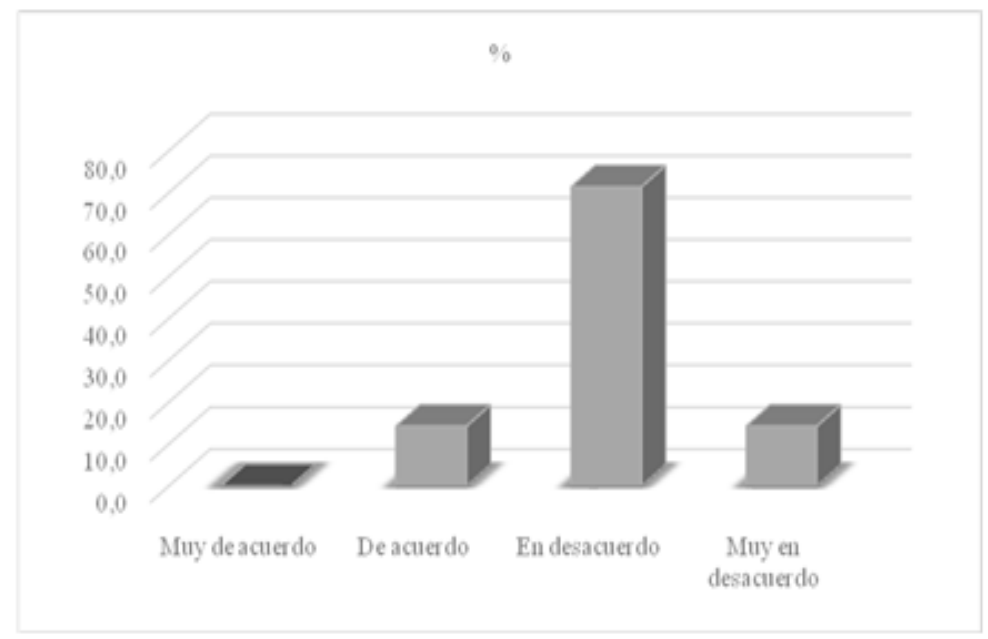

Figura 5. Pregunta a los expertos sobre la tesis: "El Estado Colombiano tiene un marco

instrumental que permita la articulación de los instrumentos de planificación sectorial en relación con el territorio de Turbo y sus condiciones ambientales".

Como caso típico de la desarticulación de política, se tiene a la Ley 388 de1997 (Congreso de Colombia, 1997), la cual plantea que las determinantes ambientales para los planes de ordenamiento territorial municipal deben centrar sus definiciones en las normas de superior jerarquía, pero no hay claridad en la definición de otros tipos de variables (por fuera de las normas de superior jerarquía), que pueden ser parte de las determinantes ambientales y que jueguen un papel importante a nivel municipal, presentándose una amplia diversidad de criterios en las Corporaciones Autónomas Regionales y dificultades en los procesos de concertación ambiental entre los municipios y las CARs, situación que evidentemente desarmoniza aún más la aplicación de política nacional colombiana en los casos concretos a nivel local.

En la siguiente tabla se hace un análisis de la articulación de los instrumentos de ordenamiento ambiental del municipio de Turbo considerando entre otros los 
soportes legales, los ecosistemas que alberga, los macroproyectos que afectan y el traslape con otros instrumentos:

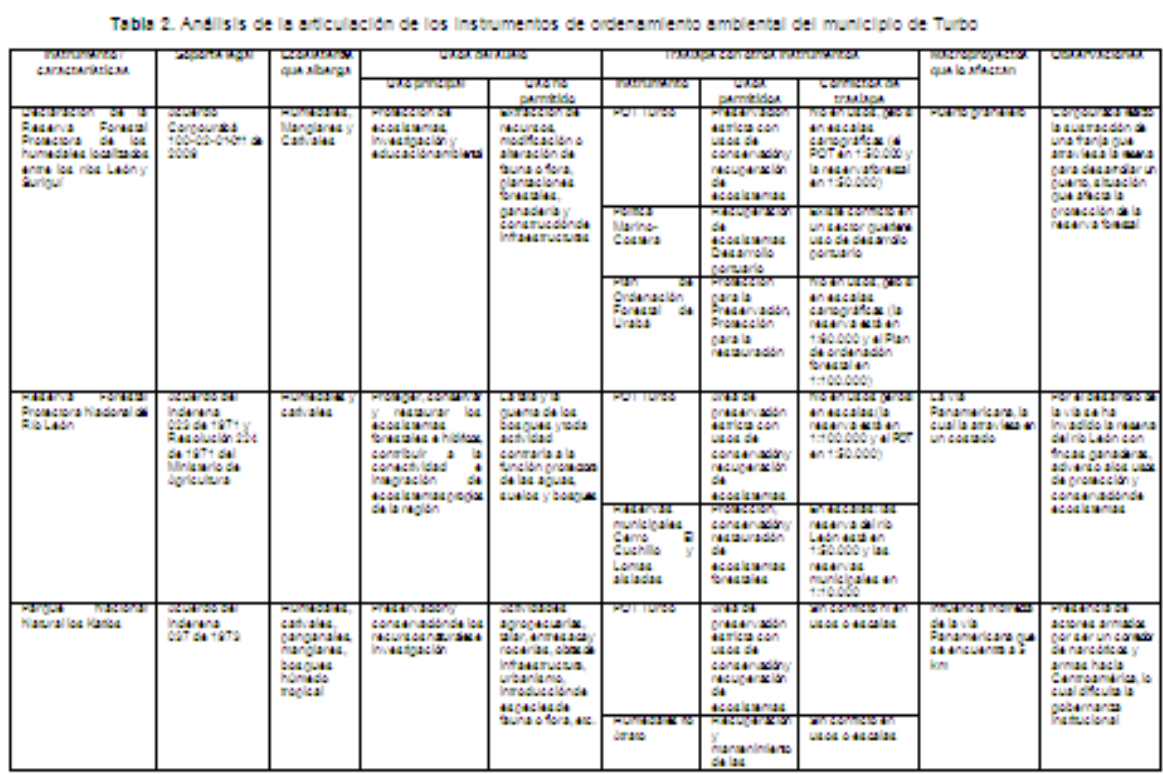

Haga clic sobre la imagen para ampliarla

Ahora bien, las políticas ambientales generadas por las autoridades del Sistema Nacional Ambiental y las formuladas por los sectores agropecuarios carecen de un enfoque territorial que reconozca las condiciones ambientales y las diferencias territoriales de las regiones del país y en concreto del municipio de Turbo, por lo cual los expertos al preguntárseles si "No hay claridad en las categorías derivadas de las legislaciones sectoriales, en cuanto al uso del suelo y frente a condiciones ambientales a su interior", el 57\% respondió "estar de acuerdo" con la apreciación y el 14\% indicó estar "muy de acuerdo" (Figura 6).

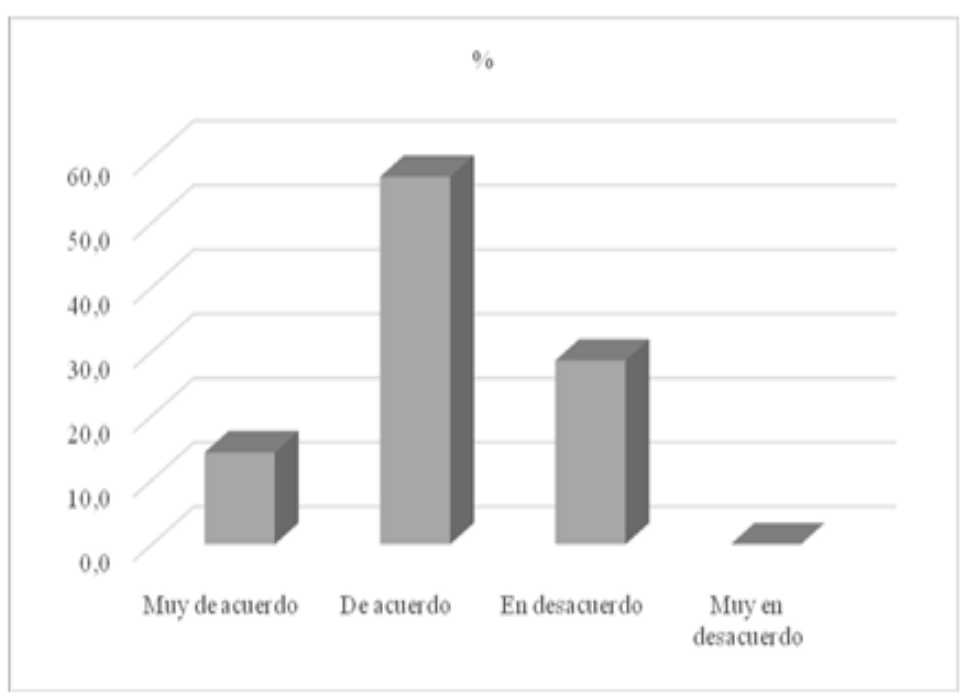

Figura 6. Pregunta a los expertos: ¿No hay claridad en las categorías derivadas de las legislaciones sectoriales, en cuanto al uso del suelo y frente a condiciones ambientales a su interior? 
En este contexto, las políticas nacionales planteadas por el Ministerio de Ambiente y Desarrollo Sostenible en materia de ordenamiento ambiental, tienen dificultades para ser armonizadas con las visiones locales (municipales), lo que genera conflictos en el territorio y se visualiza específicamente en los diferentes instrumentos de ordenamiento ambiental del municipio de Turbo. Ello porque, entre otras cosas, las políticas nacionales tienden a ser homogéneas para la totalidad de los municipios colombianos, donde no se diferencian las particularidades regionales y municipales. En este sentido, al preguntárseles a los expertos por la premisa "Las políticas de ordenamiento ambiental nacional tienden a ser homogéneas para la totalidad del territorio con baja flexibilidad al reconocimiento de las diferencias", el $71,4 \%$ respondió "estar muy de acuerdo" y el $28,6 \%$ dijo "estar de acuerdo".

Las dificultades para territorializar las políticas públicas nacionales de ordenamiento ambiental, incluso las del nivel departamental, conllevan a la aplicación de múltiples lógicas sobre el territorio del municipio de Turbo, sin poder lograr integrar territorialmente las diferentes apuestas de los agentes económicos y sociales. De allí que el POT de Turbo padece de capacidad integradora, su legitimidad a través de pactos con los sectores y actores municipales, es limitada, lo que hace difícil los procesos de planeación y actuación en temáticas relacionadas con el desarrollo sostenible.

Respecto a lo anterior, al ser consultados los expertos el $71,4 \%$ manifestaron que están "de acuerdo" con la apreciación "Dentro de los procesos de ordenamiento territorial municipales, existe profusión de categorías para el manejo del territorio, que dificultan las posibilidades de comprensión y manejo".

Para el ejercicio del ordenamiento ambiental territorial, el Estado colombiano tiene tres escalas claramente definidas: la municipal, para los planes de ordenamiento territorial (POT), la regional, definida por la jurisdicción de las autoridades ambientales regionales (las CARs) encargadas de los Planes de Gestión Ambiental Regional, los Planes de Acción Corporativo y los planes de ordenamiento y manejo de las cuencas hidrográficas (POMCAs), la Ley de ordenamiento territorial, que ha incorporado la posibilidad del ordenamiento territorial departamental.

No obstante la existencia de las tres escalas de ordenamiento ambiental, no existe un marco articulado de escalas de intervención del ordenamiento ambiental sobre el territorio en función de los objetivos de política, programáticos o de proyectos, situación que se observa en el municipio de Turbo.

Ahora bien, a través de la legislación ambiental se han desarrollado diversos instrumentos de ordenamiento y planeación ambiental (ecosistemas marinos, hídricos, humedales, paramos, entre otros), que no han sido objeto de un proceso sistemático de definición, con alcances, contenidos y objetivos diferentes a lograr, articulados a distintas escalas y metodologías y a diversos tipos de intervención. Premisa que corroboran los expertos consultados, que en un $81 \%$ consideran que no existe para el nivel local una sistematización de los diferentes instrumentos de ordenamiento ambiental y que por el contrario se da una profusión de categorías para el manejo del territorio, que dificultan las posibilidades de comprensión y manejo. 


\section{CONCLUSIONES}

- El problema principal de los instrumentos de ordenamiento ambiental de Turbo, tiene su génesis en la manera de abordar la planificación y formulación de política pública ambiental desde el Estado central, así como de dictar regulaciones, donde a nivel del SINA no existe unidad conceptual, de escala ni de enfoque en el diseño y orientación de la política pública ambiental en su relación con el territorio, lo que evidencia grietas conceptuales y formas diversas desarticuladas de planificación del desarrollo y del quehacer de lo ambiental, dando lugar con ello, a la ausencia efectiva de un ejercicio articulado de sus funciones condicionantes y determinantes de la manera de incorporar la política ambiental en la planificación sectorial del desarrollo y en general en la política pública.

- Es clave el papel que juegan las comunidades étnicas, pues todas estas figuras ambientales se definen dentro de sus territorios, en zonas que tienen una importancia económica y geoestratégica y que, debido a la actividad antrópica, genera necesariamente conflictos con los temas de protección ambiental. Se requiere así llegar a acuerdos que definan o ratifiquen una zonificación ambiental, que permita a su vez tener una cartografía unificada.

- Las diferentes zonificaciones ambientales y territoriales se cruzan sobre las mismas zonas geográficas, coincidiendo hasta seis veces diferentes tratamientos sobre la misma área. En algunas situaciones escasas dicho tratamiento coincide en mayor o menor grado con una o dos figuras ambientales, siendo la UAC Darién la figura ambiental que más trata de integrarse con otras. Pero en la mayoría no hay coincidencia clara en el tratamiento ambiental, pues si bien pueden ser tratamientos de Protección y Reserva, hay gradaciones y tratamientos diferenciales que no necesariamente serían los mismos.

- Dada esta situación de superposiciones de grandes porciones de áreas con diferentes tipos de figuras, que determinan su ordenamiento, es necesario aclarar cuáles priman sobre otras, cómo es la relación entre ellas y generar acuerdos para una zonificación única. En especial con respecto a las Consejos Comunitarios Afrocolombianos, que generan actividad antrópica sobre dichos territorios y sobre los cuales tienen autonomía.

- El desarrollo y aplicación del concepto de ordenamiento ambiental del territorio se evidencia actualmente como una tarea aplazada desde el punto de vista jurídico y de formulación de política pública, para lo cual es necesario el empoderamiento del SINA frente al ordenamiento del territorio a fin de garantizar la construcción de política.

- El rol del Ministerio de Ambiente y Desarrollo Sostenible como responsable de imprimir en las políticas sectoriales y en la planificación del desarrollo lineamientos y directrices para la adecuada inserción de la función pública del ordenamiento ambiental del territorio, supone para la institución rectora del Sistema Nacional Ambiental y de la política pública sobre medio ambiente en el país, la adopción de reglas claras que regulen y protocolicen la metodología para la valoración e inserción del ordenamiento ambiental del territorio en cada política y regulación formulada por el Ministerio desde cualquiera de los enfoques de su gestión.

- El Ministerio de Ambiente y Desarrollo Sostenible en materia de ordenamiento ambiental tiene un doble reto: de una parte, debe procurar lograr consensos con los distintos sectores de la administración pública, a efectos de lograr insertar en los procesos sectoriales de formulación de regulaciones y políticas, los lineamientos y criterios que se establezcan frente al ordenamiento del territorio, y de otra parte, debe formular unos lineamientos y directrices de tal naturaleza que 
permitan la ejecución de una política o regulación sectorial y la efectividad en el logro de los propósitos de la política de ordenamiento ambiental territorial.

\section{POTENCIAL CONFLICTO DE INTERESES}

No existe conflicto de intereses.

\section{FUENTES DE FINANCIACIÓN}

No se tuvo una fuente de financiación.

\section{REFERENCIAS}

- Astigarraga, N. (2003). El Método Delphi. Universidad de Deusto, San Sebastián. Recuperado http://www.prospectiva.eu/zaharra/Metodo_delphi.pdf

- Betancur, O. (1999). Plan de Ordenamiento Territorial. Zona centro de Urabá. Informe Socio-cultural preliminar. Medellín: Instituto de Estudios Regionales -Iner-, Centro de Investigaciones Sociales y Humanas -Cish, Universidad de Antioquia.

- Cabanzo, F. y Fracasso, L. (1997). Plan de Gestión Ambiental de Cartagena. IDEADE.

- CEPAL, 2014. Evaluaciones del desempeño ambiental en Colombia disponible en: http://www.oecd.org/env/countryreviews/Evaluacion_y_recomendacion es_Colombia.pdf .

- Comisión Tripartita. (2009). Lineamiento de Ordenamiento Territorial para Antioquia LOTA, propuesta pública para discusión, Medellín.

- Congreso de Colombia. (1993). Ley 99 de 1993. Bogotá: Imprenta Nacional.

- Congreso de Colombia. (1997). Ley 388 de 1997. Bogotá: Imprenta Nacional.

- Corpourabá. (2012). Plan de Gestión Ambiental Regional 2012-2024, Apartadó.

- Gallo, P. (2012). Aspectos conceptuales para el ordenamiento ambiental territorial. Contrato de prestación de servicios F015 DE 2012, Ministerio de Ambiente y Desarrollo Sostenible.

- Gobernación de Antioquia. (2005). Ecosistemas Estratégicos del Departamento de Antioquia. Medellín: Imprenta Departamental.

- Gobernación de Antioquia. (2008). Lineamientos y Estrategias de Manejo Integrado de la Unidad Ambiental Costera del Darién. Medellín: Imprenta Departamental.

- Gobernación de Antioquia. (2011). Anuario Estadístico de Antioquia 2010. Medellín: Imprenta Departamental.

- Gutiérrez, F. (2012). Desarrollo Sostenible o vida territorial sostenible nuevas visiones. En La educación geográfica ante los retos del siglo XXI. Bogotá: Universidad Pedagógica y Tecnológica de Colombia. ISBN 978958-57938-0-4.

- Landeta, J. (1999). El método Delphi. Barcelona: Editorial Ariel. 
- Ministerio de Ambiente, Vivienda y Desarrollo Territorial. (2011). Decreto Ley 3570. Bogotá: Imprenta Nacional.

- Ministerio de Ambiente y Desarrollo Sostenible. (2011). Política nacional para la gestión integral de la biodiversidad y sus servicios ecosistémicos. Recuperado

de https://www.siac.gov.co/documentos/DOC_Portal/DOC_Biodiversidad/0 10812_PNGIBSE_2012.pdf

- Ministerio del Interior y de Justicia. (2011). Ley Orgánica de Ordenamiento Territorial.

Recuperado de https://colaboracion.dnp.gov.co/CDT/Normatividad/CARTILLA\%2OLEY \%20ORGANICA\%20DE\%20 ORDENAMIENTO\%20TERRITORIAL.\%2028\%20nov\%20011.PDF

- Motta, V. (2005). Código de Recursos Naturales y Normas de Protección Ambiental. Bogotá: Editorial Ecode Ediciones.

- Municipio de Turbo. (2008). Agenda Ambiental Distrito Especial de Turbo.

- Municipio de Turbo. (2012). Sistema Local de Áreas Protegidas (SILAP) del municipio de Turbo, Antioquia.

- Restrepo, G. (2011). Aproximación cultural al concepto del territorio. Recuperado

de http://www.banrepcultural.org/blaavirtual/geografia/geografia/aprox.htm

- Toro, Z.Z. (2012). Territorio-lugar: Espacio de resistencia y lucha de movimientos sociales. Pacarina del Sur, 11.

1. Magíster. Maestría en Desarrollo Sostenible, Universidad de Manizales. Manizales, Colombia. Dayro3000 @yahoo.es

2. Magíster en Desarrollo Sostenible y Medio Ambiente. Docente Universidad de Manizales. Manizales, Colombia. gflorez@umanizales.edu.co

Para citar este artículo: Acevedo Ortiz, D.A. y Flórez Yepes, G.Y. (2016). Estudio de los instrumentos normativos de ordenamiento ambiental del municipio de Turbo (Antioquia), en el marco de las políticas de ordenamiento ambiental de Colombia. Revista Luna Azul, 42 , 167-184 . Recuperado de

http://200.21.104.25/lunazul//index.php?option=com_content\&view=article\&id=133 